degrees of abnormality in the ERPs. In this study, after a transient beneficial response, the cumulative effect of carbamazepine was associated with a chronic impairment of cognitive function, as measured by changes in auditory event-related potentials.

Studies of the effects of carbamazepine on auditory brainstem responses $(A B R)$ in 21 epileptic patients examined at the Institute of Clinical and Experimental Neurology, Thilisi, Republic of Georgia, demonstrated prolongation of ABR peak latencies and interpeak intervals. In addition, CBZ was associated with increases in peak latencies of middle-latency responses and slow cortical potentials. CBZ has suppressive influences on central auditory structures and the acoustic nerve. (Japaridze G et al. Epilepsia Nov/Dec 1993; $34: 1105-1109$ ).

\title{
CARBAMAZEPINE TOXICITY WITH GENERIC SUBSTITUTION
}

Two 6-year-old children with carbamazepine (CBZ) toxicity, reported from the University of Miami School of Medicine, were found to have $22 \%$ and $41 \%$ increases in serum CBZ levels after substitution of Tegretol with the generic brand, Epitol, because of insurance company policies. Adverse effects included lethargy, ataxia, slurred speech, and nystagmus. When dosage was adjusted, symptoms of toxicity resolved. (Gilman JT, Alvarez LA, Duchowny M. Carbamazepine toxicity resulting from generic substitution. Neurology Dec 1993; 43:2696-7). (Reprints: Dr Jamie Gilman, Clinical Pharmacology, Miami Children's Hospital, 6125 SW 31st Street, Miami, FL 33155).

COMMENT. Generic substitution of Tegretol has previously been associated with lowered serum levels of CBZ and seizure exacerbation. Reduced bioavailability is also reported with moisture-exposed CBZ, resulting in status epilepticus (Bell WL et al. Epilepsia Nov/Dec 1993;34:1102-4). Gilman et al have documented 2 cases of increased bioavailability with Epitol substitution, one of 4 generic carbamazepine products available in the US. In 1988, 70 million CBZ tablets were recalled because of bioinequivalence and clinical seizure exacerbation (Oles KS, Gal P. Bioequivalency revisited: Epitol versus Tegretol. Editorial. Neurology Dec 1993;43:2435-6). Factors other than generic substitution may account for significant variations in CBZ concentrations, including interlot variability, exposure of drug to excessive heat or moisture, food and drug interactions, sample timing, and patient compliance.

\section{ATTENTION DEFICIT AND COGNITIVE DISORDERS}

\section{METHYLPHENIDATE AND SLEEP PATTERNS}

The effects of methylphenidate $(0.3-0.4 \mathrm{mg} / \mathrm{kg}) \mathrm{cf}$ placebo on sleep in 10 children with ADHD are reported in a double-blind crossover study at the Bnai Zion Medical Center and the Technion-Israel Institute of Technology, Haifa, Israel. Sleep duration was significantly shorter during the drug period compared to placebo or baseline periods. The percent of quiet sleep was lower in the ADHD study group compared with controls in baseline measures, but not during methylphenidate treatment. (Tirosh E et al. Effects of methylphenidate on sleep in children with attention-deficit hyperactivity disorder. AIDC Dec 1993; 147:1313-1315). (Reprints: Dr Tirosh, Hannah Khoushy Child Development Center, Bnai Zion Medical Center, POB 4940, Haifa, Israel). 